\title{
Influence of GnRH antagonist in reproductive women on in vitro fertilization and embryo transfer in fresh cycles
}

\author{
YANG XU $^{1,2}$, YU-SONG ZHANG ${ }^{3}$, DONG-YI ZHU ${ }^{2,3}$, \\ XIANG-HONG ZHAI ${ }^{2,3}$, FENG-XIA WU ${ }^{4}$ and AN-CONG WANG ${ }^{1-3}$
}

Departments of ${ }^{1}$ Reproductive Medicine, ${ }^{2}$ Obstetrics and Gynecology and ${ }^{3}$ Imaging, Linyi People's Hospital, Linyi, Shandong 276003; ${ }^{4}$ Department of Anatomy, Shandong University, Jinan, Shandong 250012, P.R. China

Received August 6, 2018; Accepted November 27, 2018

DOI: $10.3892 /$ br.2018.1176

\begin{abstract}
The aim of the present study was to evaluate the influence of a gonadotropin-releasing hormone (GnRH) antagonist compared with a GnRH agonist on in vitro fertilization (IVF) cycle outcome in reproductive women. The characteristics of treatment and outcomes of pregnancy were retrospectively compared between the antagonist (GnRH-A, antagonist group) and agonist (GnRH-a, agonist group) regimens. The area under the curve (AUC) of receiver operating characteristic (ROC) curves was also used to evaluate whether the endometrial thickness $(\mathrm{cm})$, progesterone (P) level $(\mathrm{ng} / \mathrm{ml})$ and estradiol $\left(\mathrm{E}_{2}\right)$ level $(\mathrm{pg} / \mathrm{ml})$ on the day of human chorionic gonadotropin (hCG) administration (hCG day) had the ideal sensitivity and specificity for predicting clinical pregnancy. There were no significant differences in the baseline profiles of luteinizing hormone, $\mathrm{E}_{2}$ and $\mathrm{P}$ between the GnRH-A and GnRH-a groups $(\mathrm{P}=0.646,0.224$ and 0.119 , respectively). However age, body mass index and follicle stimulating hormone (FSH) level significantly differed between the two groups $(\mathrm{P}<0.001,=0.025$ and $<0.001$, respectively). Regarding treatment, there were significant differences in the stimulation duration (recombinant FSH days of usage), dose of gonadotrophins, $\mathrm{E}_{2}$, and $\mathrm{P}$ levels on hCG day, endometrial thickness on hCG day, mean number of total oocytes retrieved, mean number of two pronuclei oocytes, mean number of embryos available and mean number of embryos transferred (all $\mathrm{P}<0.001$ ). The rate of clinical pregnancy was lower with the GnRH antagonist than with the GnRH agonist $(\mathrm{P}<0.001)$. Additionally, the live birth rate in the GnRH-A group was significantly lower than that in the $\mathrm{GnRH}$-a group $(\mathrm{P}<0.001)$. The rate of ectopic pregnancy
\end{abstract}

Correspondence to: Professor An-Cong Wang, Department of Reproductive Medicine, Linyi People's Hospital, 27 Jiefang Road, Linyi, Shandong 276003, P.R. China

E-mail: ancongw12@163.com

Key words: controlled ovarian hyperstimulation, in vitro fertilization, gonadotropin-releasing hormone agonist/antagonist, clinical pregnancy rate did not differ significantly between the treatment groups $(\mathrm{P}=0.840)$. However, the rate of ovarian hyperstimulation syndrome (OHSS) in group GnRH-A was significantly lower than that in group $\mathrm{GnRH}-\mathrm{a}(\mathrm{P}=0.039)$. Therefore, in the present series of patients who underwent IVF embryo transfer cycles, a GnRH antagonist protocol was associated with significantly lower rates of clinical pregnancy and live birth compared with a GnRH agonist protocol; however, the rate of OHSS was significantly lower with $\mathrm{GnRH}$ antagonist compared with GnRH agonist. Furthermore, the results of the influence of endometrial thickness on clinical pregnancy, based on the ROC curve (AUC), demonstrated that the AUC was 0.553 [95\% confidence interval (CI): 0.521-0.585], and with a cutoff of $9.25 \mathrm{~cm}$, the Youden index [sensitivity-(1-specificity)] was 0.085 . The results of the influence of $E_{2}$ level on hCG day on the clinical pregnancy rate revealed an AUC of 0.613 (95\% CI: $0.581-0.644$ ), and with a cutoff of $1,520 \mathrm{pg} / \mathrm{ml}$, the Youden index was 0.184 . The results of the influence of $\mathrm{P}$ level on hCG day $(\mathrm{ng} / \mathrm{ml})$ on the clinical pregnancy rate revealed an AUC of 0.526 (95\% CI: $0.494-0.558$ ), and with a cutoff of $0.415 \mathrm{ng} / \mathrm{ml}$, the Youden index was 0.061 . These results of the ROC curve analyses demonstrated that neither the endometrial thickness nor the $\mathrm{E}_{2}$ and $\mathrm{P}$ levels on hCG day had the ideal sensitivity or specificity for predicting clinical pregnancy.

\section{Introduction}

Luteinizing hormone ( $\mathrm{LH})$ is essential for normal follicular development and oocyte maturation. It is established that an essential part of ovarian stimulation in in vitro fertilization (IVF) is to prevent premature luteinization (1). There are two protocols for pituitary desensitization, either lengthening daily administration of gonadotropin-releasing hormone (GnRH) agonists, or immediately blocking the secretion of the pituitary LH with GnRH antagonist. Each procedure can effectively block premature LH surges $(2,3)$.

In recent decades, GnRH antagonists have been applied in clinical practice for ovarian stimulation protocols in IVF. Compared with GnRH agonists, GnRH antagonists are advantageous in that they require a shorter duration of treatment, a shorter duration for follicle stimulating hormone (FSH) stimulation, and are associated with a relatively lower risk of ovarian hyperstimulation syndrome (OHSS) (4). One particular 
advantage in the prevention of OHSS with the GnRH antagonist protocol is that $\mathrm{GnRH}$ agonists act as trigger, inducing a transient endogenous LH surge, rather than a human chorionic gonadotropin (hCG) trigger that induces an extended exogenous LH surge, particularly in patients with an expected or known high response $(5,6)$.

At present, there remains to be debate regarding the safety and efficacy of GnRH antagonists and agonists for IVF embryo transfer (IVF-ET). Individualization of ovarian stimulation for IVF is increasingly common to tailor the stimulation protocol to the patient's specific preconditions. To determine the advantages and disadvantages of antagonist and agonist regimens, investigation is required in specific classifications of IVF patients, such as in patients with diminished ovarian reserve, in the general population, and in patients with polycystic ovary syndrome (PCOS). This could be relevant as these women may be particularly different in ovarian response, particularly in relation to agonists and antagonists. It has been reported that in general IVF patients, ongoing pregnancy rate was significantly lower in the antagonist group compared with in the agonist group (7). By contrast, in women with PCOS and in women with poor ovarian response, there was no significant difference in ongoing pregnancy between the two groups (7).

Therefore, the aim of the present study was to evaluate the influence of GnRH antagonist compared with GnRH agonist on IVF cycle outcome in patients in the IVF unit of Linyi People's Hospital (Linyi, China). Furthermore, the area under the curve (AUC) of receiver operating characteristic (ROC) curves was used to evaluate whether the endometrial thickness, estradiol $\left(\mathrm{E}_{2}\right)$ level and progesterone $(\mathrm{P})$ level on the day of hCG administration (hCG day) had the ideal sensitivity and specificity for predicting a clinical pregnancy in the fresh ET cycle. Overall the results were hoped to aid reproductive specialists indecision-making for infertile patients.

\section{Materials and methods}

Patients. The computerized files (clinical medicine reproductive management system) of the patients who were admitted to the IVF unit of Linyi People's Hospital from January 31, 2013 to December 31, 2016, all of whom had reached the ET step, were retrospectively reviewed. The study was approved by the Ethics Committee of Linyi People's Hospital. Patients with primary or secondary infertility were included in the study, while those with high or low ovarian reserve $(8,9)$ were excluded. All of the patients underwent controlled ovarian hyperstimulation using either the midluteal long GnRH agonist [triptorelin; Ferring $\mathrm{GmbH}$, Kiel, Germany; $0.1 \mathrm{mg}$ daily, subcutaneously (SC)] suppressive protocol (agonist group, group GnRH-a) or the multiple-dose fixed GnRH antagonist [ganirelix (ganirelix acetate); Merck-Serono, Ltd., Aubonne, Switzerland; $0.25 \mathrm{mg}$ daily, SC] protocol (antagonist group, group GnRH-A).

The selection of the type of analog to be used was the decision of the treating physicians. Data on the baseline characteristics of patients in the agonist and antagonist groups were collected from the files (Table I). ELISA kits were used to measure serum $\mathrm{E}_{2}$ (pg/ml; cat. no. 10491445), P (ng/ml; cat. no. 01586287), FSH (cat. no. 01360521) and LH (UI/1; cat. no. 02212941; all from Siemens AG, Munich, Germany).
Estimated ROC curves for the performance of endometrial thickness and $\mathrm{E}_{2}$ and $\mathrm{P}$ levels on the hCG day in the prediction of clinical pregnancy were also established.

All of the patients underwent baseline transvaginal sonography on day 2 or 3 of the menstrual cycle to check the antral follicle count and the thickness of the endometrium $(\mathrm{cm})$. The protocol for agonist and antagonist treatments were as reported previously (10). Outcomes including the mean number of total oocytes retrieved, the mean number of two pronuclei (2PN) oocytes, the mean number of embryos available, the mean number of embryos transferred and live birth rate (11-13) were recorded.

The results of the influence of endometrial thickness, $\mathrm{P}$ levels and $\mathrm{E}_{2}$ levels on clinical pregnancy, based on individual ROC curves (AUCs) and the respective Youden index [sensitivity-(1-specificity)] (14), were used to demonstrate sensitivity and specificity in predicting clinical pregnancy.

Statistical analysis. SPSS 23.0 software for Windows (IBM Corp., Armonk, NY, USA) was used for statistical analysis. The data were reported as the mean \pm standard deviation. Differences in the variables between the two groups were statistically analyzed using Student's t-test or Wilcoxon-Mann-Whitney test and Pearson's $\chi^{2}$ test at a two-sided significance level of 0.05 . In the present study, the AUC of ROC curves was used to evaluate whether the endometrial thickness and $\mathrm{E}_{2}$ and $\mathrm{P}$ levels on the hCG day had high sensitivity and specificity for predicting clinical pregnancy.

\section{Results}

Baseline characteristics and clinical outcomes in the GnRH A and GnRH a groups on IVF. A total of 1,231 fresh cycles of reproductive women with normal ovarian reserve were evaluated: 577 fresh cycles in the GnRH-A group and 654 fresh cycles in the GnRH-a group. There were significant differences in the baseline parameters of age, body mass index (BMI) and FSH basal hormone profile between the GnRH-A and GnRH-a groups (Table I). The mean age in years was $36.08 \pm 5.40$ for the GnRH-A group and $31.51 \pm 4.57$ for the GnRH-a group $(\mathrm{P}<0.001)$, and the mean BMI was 24.06 \pm 3.05 for the GnRH-A group and $23.65 \pm 3.23$ for the $\mathrm{GnRH}$-a group $(\mathrm{P}=0.025)$. The mean basal hormone profile of $\mathrm{FSH}$ was $8.23 \pm 2.63 \mathrm{UI} / 1$ for the GnRH-A group and 6.90 $1.88 \mathrm{UI} / 1$ for the $\mathrm{GnRH}$-a group $(\mathrm{P}<0.001)$. Meanwhile, the LH level did not differ significantly between the groups: the mean LH level was $4.25 \pm 2.35 \mathrm{UI} / 1$ for the GnRH-A group and 4.38 $\pm 2.47 \mathrm{UI} / 1$ for the $\mathrm{GnRH}$-a group $(\mathrm{P}=0.646)$. Additionally, mean $\mathrm{E}_{2}$ level was $47.49 \pm 19.50 \mathrm{pg} / \mathrm{ml}$ for the GnRH-A group and $44.19 \pm 18.43 \mathrm{pg} / \mathrm{ml}$ for the $\mathrm{GnRH}-\mathrm{a}$ group $(\mathrm{P}=0.224)$, and the mean $\mathrm{P}$ level was $0.44 \pm 0.26 \mathrm{ng} / \mathrm{ml}$ for the GnRH-A group and $0.42 \pm 0.22 \mathrm{ng} / \mathrm{ml}$ for the $\mathrm{GnRH}-\mathrm{a}$ group $(\mathrm{P}=0.119$; Table I).

When comparing the GnRH-A and GnRH-a groups on treatment parameters, there were significant differences in the stimulation duration ( $\mathrm{rFSH}$ days of usage: $9.26 \pm 1.60$ vs. $10.54 \pm 1.62$, respectively, $\mathrm{P}<0.001$ ), dose of gonadotrophins on hCG day $(2,021.53 \pm 590.87$ vs. $2,279.28 \pm 553.46 \mathrm{IU}$, respectively, $\mathrm{P}<0.001)$, the endometrial thickness on $\mathrm{hCG}$ day $(9.93 \pm 2.26$ vs. $10.89 \pm 2.42 \mathrm{~cm}$, respectively, $\mathrm{P}<0.001)$, the $\mathrm{E}_{2}$ level on hCG day $(1,516.58 \pm 955.91$ vs. $3,049.90 \pm 1,250.36 \mathrm{pg} / \mathrm{ml}$, 
Table I. Baseline characteristics of patients in the GnRH-A and GnRH-a groups.

\begin{tabular}{lccr}
\hline Variable & GnRH-A group (n=557 cycles) & GnRH-a group (n=654 cycles) & P-value \\
\hline Age, years & $36.06 \pm 5.41$ & $31.51 \pm 4.57$ & $<0.001$ \\
BMI, kg/m ${ }^{2}$ & $24.06 \pm 3.05$ & $23.65 \pm 3.23$ & 0.025 \\
FSH, UI/l & $8.23 \pm 2.63$ & $6.90 \pm 1.88$ & $<0.001$ \\
LH, UI/l & $4.25 \pm 2.35$ & $4.38 \pm 2.47$ & $0.646^{\mathrm{a}}$ \\
$\mathrm{E}_{2}, \mathrm{pg} / \mathrm{ml}$ & $47.49 \pm 19.50$ & $44.19 \pm 18.43$ & $0.224^{\mathrm{a}}$ \\
$\mathrm{P}, \mathrm{ng} / \mathrm{ml}$ & $0.44 \pm 0.26$ & $0.42 \pm 0.22$ & 0.119
\end{tabular}

${ }^{a}$ Mann-Whitney U test. Other data were analyzed by t-test. GnRH-A/a, gonadotropin-releasing hormone-antagonist/agonist; BMI, body mass index; FSH, follicle stimulating hormone; $\mathrm{LH}$, luteinizing hormone; $\mathrm{E}_{2}$, estradiol; P, progesterone.

Table II. Comparison of the groups on stimulation characteristics and outcomes in in vitro fertilization.

\begin{tabular}{|c|c|c|c|}
\hline Characteristics & GnRH-A group ( $\mathrm{n}=577$ cycles) & GnRH-a group ( $n=654$ cycles) & P-value \\
\hline Stimulation duration, days & $9.26 \pm 1.60$ & $10.54 \pm 1.62$ & $<0.001$ \\
\hline Dose of gonadotrophins, IU & $2,021.53 \pm 590.87$ & $2,279.28 \pm 553.46$ & $<0.001$ \\
\hline $\mathrm{E}_{2}$ on hCG day, pg/ml & $1,516.58 \pm 955.91$ & $3,049.90 \pm 1250.36$ & $<0.001$ \\
\hline $\mathrm{P}$ on hCG day, ng/ml & $0.61 \pm 0.30$ & $0.71 \pm 0.27$ & $<0.001$ \\
\hline $\mathrm{EM}$ on hCG day, $\mathrm{cm}$ & $9.93 \pm 2.26$ & $10.89 \pm 2.42$ & $<0.001$ \\
\hline Mean no. of total oocytes retrieved & $5.31 \pm 3.26$ & $8.63 \pm 3.73$ & $<0.001$ \\
\hline Mean no. of 2PN oocytes & $3.71 \pm 2.66$ & $6.21 \pm 4.21$ & $<0.001^{\mathrm{a}}$ \\
\hline Mean no. of embryos available & $2.54 \pm 1.71$ & $3.76 \pm 2.24$ & $<0.001^{\mathrm{a}}$ \\
\hline Mean no. of embryos transferred & $1.74 \pm 0.69$ & $1.92 \pm 0.40$ & $<0.001^{\mathrm{a}}$ \\
\hline Clinical pregnancy rate, n (\%) & $185(32.06)$ & $379(57.95)$ & $<0.001$ \\
\hline Ectopic pregnancy rate, n (\%) & $9 / 185(4.86)$ & $17 / 379(4.49)$ & 0.840 \\
\hline Live birth rate, $\mathrm{n}(\%)$ & $144(24.96)$ & $303(46.33)$ & $<0.001$ \\
\hline OHSS rate, $\mathrm{n}(\%)$ & $7(1.21)$ & $19(2.91)$ & 0.039 \\
\hline
\end{tabular}

Live birth was defined as successful delivery of a live-born infant. ${ }^{a}$ Mann-Whitney U test. Other data were analyzed by t-test. GnRH-A/a, gonadotropin-releasing hormone-antagonist/agonist; hCG, human chorionic gonadotropin; $\mathrm{E}_{2}$, estradiol; P, progesterone; EM, endometrial thickness; 2PN, two pronuclei; OHSS, ovarian hyperstimulation syndrome.

respectively, $\mathrm{P}<0.001)$, the $\mathrm{P}$ level on $\mathrm{hCG}$ day $(0.61 \pm 0.30$ vs. $0.71 \pm 0.27 \mathrm{ng} / \mathrm{ml}$, respectively, $\mathrm{P}<0.001)$, the mean number of total oocytes retrieved $(5.31 \pm 3.26$ vs. $8.63 \pm 3.73$, respectively, $\mathrm{P}<0.001)$, the mean number of two pronuclei $(2 \mathrm{PN})$ oocytes $(3.71 \pm 2.66$ vs. $6.21 \pm 4.21$, respectively, $\mathrm{P}<0.001)$, the mean number of embryos available $(2.54 \pm 1.71$ vs. $3.76 \pm 2.24$, respectively, $\mathrm{P}<0.001)$ and the mean number of embryos transferred $(1.74 \pm 0.69$ vs. $1.92 \pm 0.40$, respectively, $\mathrm{P}<0.001$; Table II).

There was significant differences in the pregnancy outcomes between the GnRH-A and GnRH-a groups, including in clinical pregnancy rate [185 (32.06\%) vs. $379(57.95 \%)$, respectively, $\mathrm{P}<0.001)$. The group of patients who received GnRH-A exhibited a lower rate of live births compared with that recorded for the GnRH-a group [144 (24.96\%) vs. 303 $(46.33 \%), \mathrm{P}<0.001]$ and lower OHSS rate [7 (1.21\%) vs. 19 $(2.91 \%), P=0.039]$. However, there was no significant difference in the outcome of ectopic pregnancy rate [9 $(4.86 \%)$ vs. $17(4.49 \%), \mathrm{P}=0.840]$.
Estimated ROC curves for the performance of endometrial thickness and $E_{2}$ and $P$ levels on the $h C G$ day in the prediction of clinical pregnancy. The plots of the sensitivity-specificity combinations in an ROC space are presented in Figs. 1-3. The results of the influence of endometrial thickness on clinical pregnancy, based on the ROC curve (AUC), demonstrated that the AUC was 0.553 [95\% confidence interval (CI): 0.521-0.585], and with a cutoff of $9.25 \mathrm{~cm}$, the sensitivity was $68.6 \%$ and the specificity was $39.9 \%$. Additionally, the Youden index was 0.085 (Fig. 1). The results of the influence of the $\mathrm{E}_{2}$ level on hCG day on the clinical pregnancy rate, from the ROC curve (AUC), demonstrated that the AUC was 0.613 (95\% CI: 0.581-0.644), and with a cutoff of $1,520 \mathrm{pg} / \mathrm{ml}$, the sensitivity was $78.4 \%$ and the specificity was $40.0 \%$. Additionally, the Youden index was 0.184 (Fig. 2). The results of the influence of the P level on hCG day (ng/ml) on the clinical pregnancy rate, based on the ROC (AUC), showed that the AUC was 0.526 (95\% CI: $0.494-0.558)$, and with a cutoff of $0.415 \mathrm{ng} / \mathrm{ml}$, the sensitivity was $82.6 \%$ and the specificity was $23.5 \%$. Additionally, the 


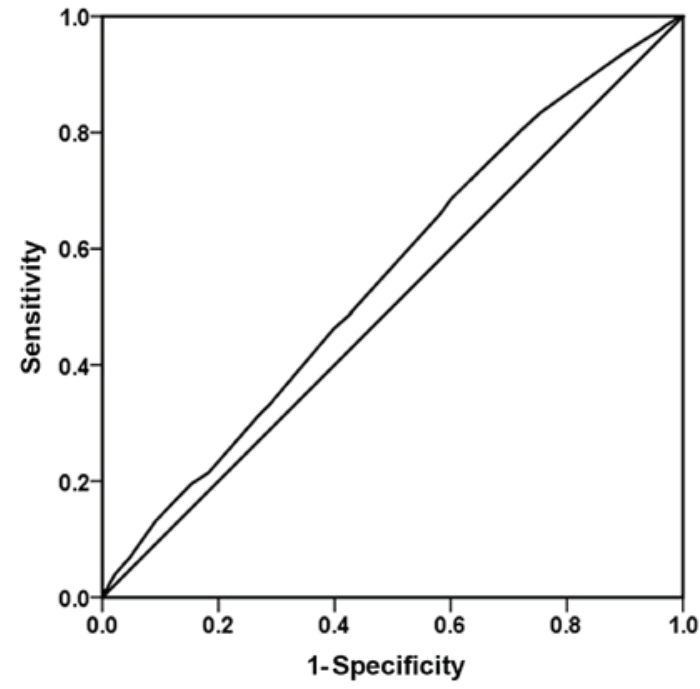

Figure 1. Estimated receiver operating characteristic curve and sensitivity-specificity points for the performance of endometrial thickness in predicting clinical pregnancy.

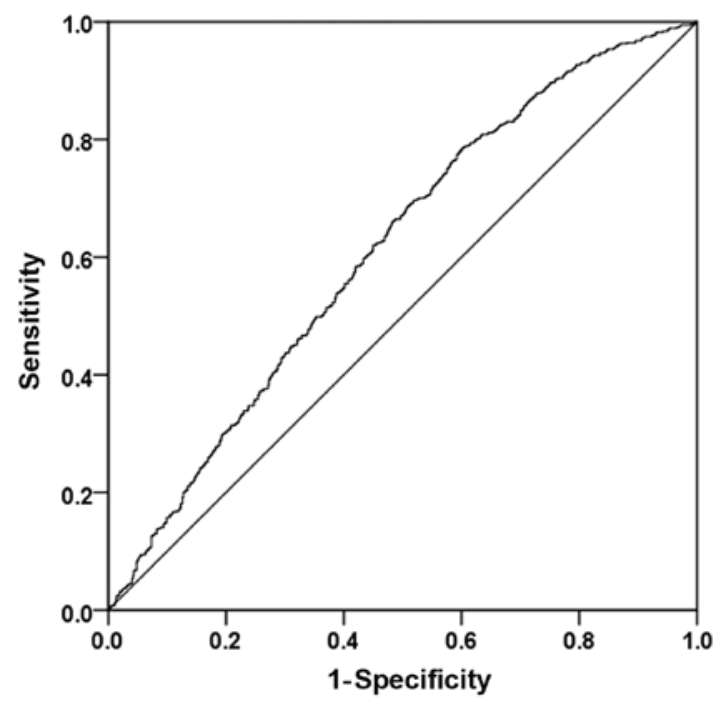

Figure 2. Estimated receiver operating characteristic curve and sensitivity-specificity points for the performance of estradiol level $(\mathrm{pg} / \mathrm{ml})$ on the day of human chorionic gonadotropin administration in predicting clinical pregnancy.

Youden index was 0.061 (Fig. 3). These results of the ROC curves demonstrated that neither the endometrial thickness on day of ET nor the $\mathrm{E}_{2}$ and P levels on hCG day had the best sensitivity and specificity for predicting the clinical pregnancy.

\section{Discussion}

The present study compared the efficacy of two different ovarian stimulation protocols (GnRH antagonist vs. GnRH agonist) during IVF treatment in reproductive normal responder women. It has been reported that overall GnRH antagonists do not compromise effectiveness and significantly prevent OHSS (15). When antagonists have been used in patients with normal ovarian function, the clinical pregnancy rate has been reported as lower than that for the agonist

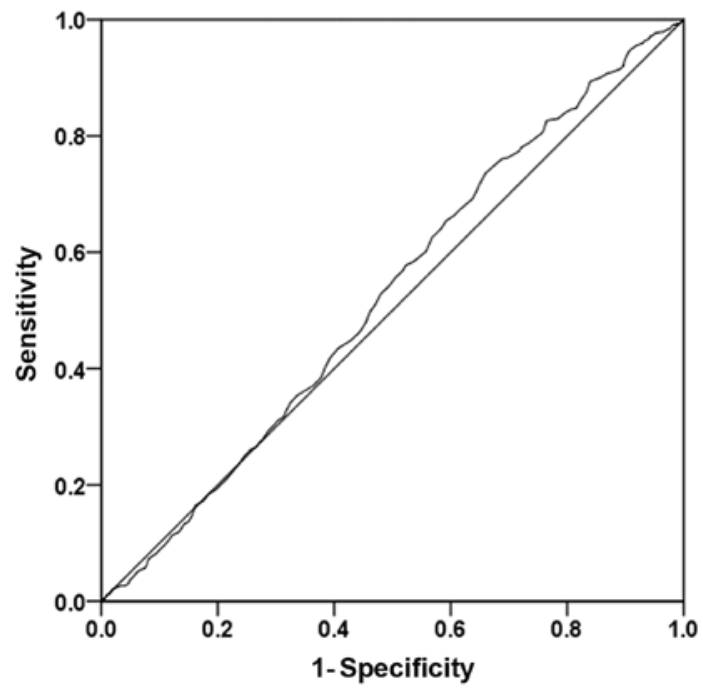

Figure 3. Estimated receiver operating characteristic curve and sensitivity-specificity points for the performance of progesterone level (ng/ml) on the day of human chorionic gonadotropin administration in predicting clinical pregnancy.

protocol, potentially due to the antagonist exerting a negative effect directly or indirectly on endometrial receptivity, for instance by promoting premature maturation of the endometrium (16-18). It is undesirable that in the present study, there were significant differences in the baseline parameters of age, BMI and FSH hormone profile between the GnRH-A and GnRH-a groups, which differed from similar previous study by our group on polycystic ovary syndrome (10).

Cumulative studies have confirmed that an association exists between age and fertility; ovarian aging and reduced ovarian reserve are considered critical factors in determining IVF outcomes (19-21). Here, the mean age of the GnRH-a group was significantly lower than that of the GnRH-A group, which may indicate that the groups were not representative of a single general population. This would limit the conclusions of the present study.

It is generally known that extremely high levels of $E_{2}$ are harmful to embryo condition and endometrial receptivity, which may impact on embryo implantation. From the above data, in the present study, there were significantly higher $E_{2}$ levels on hCG day in the GnRH-a group compared with in the GnRH-A group. The GnRH-a protocol was also associated with significantly higher rates of clinical pregnancy and live birth compared with those rates in the GnRH-A group. Nevertheless, there was no significant difference in ectopic pregnancy rate between the groups.

Implantation is necessary for successful pregnancy and requires adequate endometrial receptivity, which is among the most important factors in predicting pregnancy following IVF-ET. In particular the endometrial thickness has been documented as an individual indicator for endometrial receptivity $(22,23)$. Previous study observed that the rates of clinical pregnancy and live birth were markedly lower in patients with an endometrium thickness of $\leq 7 \mathrm{~mm}$, compared within those patients with an endometrium thickness of $>7 \mathrm{~mm}$; however, there was no significant difference in the rates of clinical pregnancy and implantation with endometrial thicknesses in 
the $8-14 \mathrm{~mm}$ range (24). In the present study, it was identified that the endometrial thickness on the $\mathrm{hCG}$ day ranged from 5.5 to $18.5 \mathrm{~mm}$, and the group of patients who received the GnRH-A regimen exhibited significantly reduced thickness compared with those receiving the GnRH-a regimen. The influence of the endometrial thickness on the clinical pregnancy rate was also evaluated via ROC curve analysis. The results of the influence of the endometrial thickness (EM) on the clinical pregnancy, using the ROC (AUC), showed that the EM was a poor predictor of clinical pregnancy. At present, no conclusive cut-off value for the endometrial thickness has been established. Additionally in the current study, the results of the ROC curves demonstrated that neither $\mathrm{E}_{2}$ nor $\mathrm{P}$ level on hCG day had the best sensitivity and specificity for predicting clinical pregnancy. Further larger studies should be performed to elucidate the contribution of the endometrial thickness and hormone levels on the day of hCG administration to clinical pregnancy rate in patients receiving $\mathrm{GnRH}$ antagonist or agonist.

In conclusion, in the present series of women undergoing IVF-ET cycles, GnRH antagonist decreased oocyte retrieval, oocyte cleavage, the number of embryos available and the potential for embryo transfer. Furthermore, compared with the agonist regimen, the rates of clinical pregnancy and live birth with the antagonist regimen were significantly reduced. However, the risk of OHSS was lower with the GnRH antagonist. In the present study, the results of the ROC curves demonstrated that neither $\mathrm{E}_{2}$ nor $\mathrm{P}$ level on the day of hCG administration had ideal sensitivity or specificity for predicting clinical pregnancy, in accordance with our previous study (10). Therefore, it appears that in predicting clinical pregnancy rate, the endometrial thickness and estradiol and progesterone levels are not the ideal predictors. It may be that embryo quality itself has a greater impact on pregnancy outcomes. Nonetheless, more in-depth studies are required to confirm the predictors of clinical pregnancy.

\section{Acknowledgements}

Not applicable.

\section{Funding}

The present study was supported by the National Natural Science Fund of China (grant no. 81501620), the Shandong Provincial Natural Science Foundation of China (grant nos. ZR2014HP026 and ZR2016HP37) and the Shandong Provincial Medical and Health Science and Technology Development Project (grant no. 2015WS0377).

\section{Availability of data and materials}

The datasets used and/or analyzed in the current study are available from the corresponding author on reasonable request.

\section{Authors' contributions}

ACW was principally responsible for conception and design of the study. YX and YSZ participated in the acquisition of the reported data. DYZ and XHZ participated in the processing and interpretation of the reported data. FXW contributed to the writing and revising of the manuscript. All authors read and approved the final manuscript to be published.

\section{Ethics approval and consent to participate}

Written informed consent was obtained from all participants. The study was approved by the Ethics Committee of Linyi People's Hospital [approval no. (2017) IRB NO. (0012)].

\section{Patient consent for publication}

Informed consent was obtained for publication of the participants' data.

\section{Competing interests}

The authors declare that they have no competing interests.

\section{References}

1. Lévy DP, Navarro JM, Schattman GL, Davis OK and RosenwaksZ: The role of LH in ovarian stimulation: exogenous LH: let's design the future. Hum Reprod 15: 2258-2265, 2000.

2. Huirne JA, van Loenen AC, Schats R, McDonnell J, Hompes PG, Schoemaker J, Homburg R and Lambalk CB: Dose-finding study of daily gonadotropin-releasing hormone $(\mathrm{GnRH})$ antagonist for the prevention of premature luteinizing hormone surges in IVF/ICSI patients: Antide and hormone levels. Hum Reprod 19: 2206-2215, 2004.

3. Huirne JA, van Loenen AC, Schats R, McDonnell J, Hompes PG, Schoemaker J, Homburg R and Lambalk CB: Dose-finding study of daily $\mathrm{GnRH}$ antagonist for the prevention of premature $\mathrm{LH}$ surges in IVF/ICSI patients: Optimal changes in LH and progesterone for clinical pregnancy. Hum Reprod 20: 359-367, 2005.

4. Weiss JM, Ludwig M, Ortmann O and Diedrich K: GnRH antagonists in the treatment of infertility. Ann Med 35: 512-522, 2003.

5. Raju GA, Chavan R, Deenadayal M, Gunasheela D, Gutgutia R, Haripriya G, Govindarajan M, Patel NH and Patki AS: Luteinizing hormone and follicle stimulating hormone synergy: A review of role in controlled ovarian hyper-stimulation. J Hum Reprod Sci 6: 227-234, 2013.

6. Serafini P, Yadid I, Motta EL, Alegretti JR, Fioravanti J and Coslovsky M: Ovarian stimulation with daily late follicular phase administration of low-dose human chorionic gonadotropin for in vitro fertilization: A prospective, randomized trial. Fertil Steril 86: 830-838, 2006.

7. Lambalk CB, Banga FR, Huirne JA, Toftager M, Pinborg A, Homburg R, van der Veen F and van Wely M: GnRH antagonist versus long agonist protocols in IVF: A systematic review and meta-analysis accounting for patient type. Hum Reprod Update 23: 560-579, 2017.

8. te Velde ER and Pearson PL: The variability of female reproductive ageing. Hum Reprod Update 8: 141-154, 2002.

9. Loutradis D, Drakakis P, Vomvolaki E and Antsaklis A: Different ovarian stimulation protocols for women with diminished ovarian reserve. J Assist Reprod Genet 24: 597-611, 2007.

10. Zhai XH, Zhang P, Wu FX, Wang AC and Liu PS: GnRH antagonist for patients with polycystic ovary syndrome undergoing controlled ovarian hyperstimulation for in vitro fertilization and embryo transfer in fresh cycles. Exp Ther Med 13: 3097-3102, 2017.

11. Edwards RG, Bavister BD and Steptoe PC: Early stages of fertilization in vitro of human oocytes matured in vitro. Nature 221: 632-635, 1969.

12. Lopata A, McMaster R, McBain JC and Johnston WI: In-vitro fertilization of preovulatory human eggs. J Reprod Fertil 52: 339-342, 1978.

13. Younis JS, Radin O, Mirsky N, Izhaki I, Majara T, Bar-ami S and Ben-ami M: First polar body and nucleolar precursor body morphology is related to the ovarian reserve of infertile women. Reprod Biomed Online 16: 851-858, 2008. 
14. Bertran EA, Berlie HD, Taylor A, Divine G and Jaber LA: Diagnostic performance of HbA1c for diabetes in Arab vs. European populations: A systematic review and meta-analysis. Diabet Med 34: 156-166, 2017.

15. Al-Inany HG, Youssef MA, Ayeleke RO, Brown J, Lam WS and Broekmans FJ: Gonadotrophin-releasing hormone antagonists for assisted reproductive technology. Cochrane Database Syst Rev 4: CD001750, 2016.

16. Olivennes F, Cunha-Filho JS, Fanchin R, Bouchard P and Frydman R: The use of GnRH antagonists in ovarian stimulation. Hum Reprod Update 8: 279-290, 2002.

17. Rackow BW, Kliman HJ and Taylor HS: GnRH antagonists may affect endometrial receptivity. Fertil Steril 89: 1234-1239, 2008.

18. Kolibianakis E, Bourgain C, Albano C, Osmanagaoglu K, Smitz J, Van Steirteghem A and Devroey P: Effect of ovarian stimulation with recombinant follicle-stimulating hormone, gonadotropin releasing hormone antagonists, and human chorionic gonadotropin on endometrial maturation on the day of oocyte pick-up. Fertil Steril 78: 1025-1029, 2002.

19. Honnma H, Baba T, Sasaki M, Hashiba Y, Oguri H, Fukunaga T, Endo T and Asada Y: Different ovarian response by age in an anti-Müllerian hormone-matched group undergoing in vitro fertilization. J Assist Reprod Genet 29: 117-125, 2012.
20. Nelson SM, Telfer EE and Anderson RA: The ageing ovary and uterus: New biological insights. Hum Reprod Update 19: 67-83, 2013.

21. Bleil ME, Adler NE, Pasch LA, Sternfeld B, Gregorich SE, Rosen MP and Cedars MI: Psychological stress and reproductive aging among pre-menopausal women. Hum Reprod 27: 2720-2728, 2012.

22. Friedler S, Schenker JG, Herman A and Lewin A; FriedlerS: The role of ultrasonography in the evaluation of endometrial receptivity following assisted reproductive treatments: A critical review. Hum Reprod Update 2: 323-335, 1996.

23. Dekel N, Gnainsky Y, Granot I and Mor G: Inflammation and implantation. Am J Reprod Immunol 63: 17-21, 2010.

24. Yoeli R, Ashkenazi J, Orvieto R, Shelef M, Kaplan B and Bar-Hava I: Significance of increased endometrial thickness in assisted reproduction technology treatments. J Assist Reprod Genet 21: 285-289, 2004. 